\title{
A Diffusion Tensor Imaging Study on White Matter Abnormalities in Patients with Type 2 Diabetes Using Tract-Based Spatial Statistics
}

\author{
(D). Xiong, (D)Y. Sui, (D). Xu, (D) Q. Zhang, (D)M.M. Karaman, (D). Cai, (D)T.M. Anderson, (D)W. Zhu, (D) J. Wang, and (D)X.J. Zhou
} O- $\equiv$

\begin{abstract}
BACKGROUND AND PURPOSE: Patients with type 2 diabetes mellitus have considerably higher risk of developing cognitive impairment and dementia. WM changes in these patients have been reported. Our aim was to demonstrate that gradual and continuous WM change and the associated cognitive decline in patients with type 2 diabetes mellitus can be captured by DTI parameters, which can be used to complement neuropsychological test scores in identifying patients with type 2 diabetes mellitus with and without mild cognitive impairment.
\end{abstract}

MATERIALS AND METHODS: Forty-two patients with type 2 diabetes mellitus, divided into a group with mild cognitive impairment $(n=$ 20) and a group with normal cognition ( $n=22)$, were enrolled with age-, sex-, and education-matched healthy controls $(n=26)$. 3T DTI followed by Tract-Based Spatial Statistics analysis was used to investigate the differences in fractional anisotropy, mean diffusivity, axial diffusivity $\left(\lambda_{1}\right)$, and radial diffusivity $\left(\lambda_{23}\right)$ among the groups. A receiver operating characteristic analysis assessed the performance of DTI parameters for separating the 2 groups with type 2 diabetes mellitus.

RESULTS: The whole-brain Tract-Based Spatial Statistics analysis revealed that $7.3 \%$ and $24.9 \%$ of the WM exhibited decreased fractional anisotropy and increased mean diffusivity $(P<.05)$, respectively, between the diabetes mellitus with mild cognitive impairment and the diabetes mellitus with normal cognition groups, while considerably larger WM regions showed fractional anisotropy (36.6\%) and mean diffusivity (58.8\%) changes between the diabetes mellitus with mild cognitive impairment and the healthy control groups. These changes were caused primarily by an elevated radial diffusivity observed in the patients with diabetes mellitus with mild cognitive impairment. Radial diffusivity also exhibited subtle but statistically significant changes between the diabetes mellitus with normal cognition and the healthy control groups. Analyses on individual fiber tracts showed pronounced fractional anisotropy reduction and mean diffusivity elevation in regions related to cognitive functions. The receiver operating characteristic analysis on the right cingulum (hippocampus) showed that fractional anisotropy produced a larger area under the curve (0.832) than mean diffusivity (0.753) for separating mild cognitive impairment from normal cognition among patients with type 2 diabetes mellitus. When fractional anisotropy was combined with mean diffusivity, the area under the curve was further improved to 0.857 .

CONCLUSIONS: DTI parameters can show a substantial difference between patients with type 2 diabetes mellitus with and without mild cognitive impairment, suggesting their potential use as an imaging marker for detecting cognitive decline in patients with type 2 diabetes mellitus. More important, DTI parameters may capture gradual and continuous WM changes that can be associated with early stages of cognitive decline in patients with type 2 diabetes mellitus before they can be diagnosed clinically by using conventional neuropsychological tests.

ABBREVIATIONS: $\mathrm{DM}-\mathrm{MCI}=$ diabetes mellitus with mild cognitive impairment; $\mathrm{DM}-\mathrm{NC}=$ diabetes mellitus with normal cognition; $\mathrm{FA}=$ fractional anisotropy; $\mathrm{HC}=$ healthy control; $\mathrm{MCl}=$ mild cognitive impairment; $\mathrm{MD}=$ mean diffusivity; TBSS = Tract-Based Spatial Statistics; T2DM = type 2 diabetes mellitus; $\mathrm{ROC}=$ receiver operating characteristic

T ype 2 diabetes mellitus (T2DM) is a prevalent disease that affects $>360$ million people worldwide and is projected to rise to 552 million cases by 2030. ${ }^{1}$ This metabolic disease can affect a number of

Received July 14, 2015; accepted after revision January 7, 2016

From the Departments of Radiology (Y.X., W.Z.) and Neurology (Q.Z.), Tongji Hospital, and Department of Pathophysiology (Z.X., J.W.), Tongji Medical College, Huazhong University of Science and Technology, Wuhan, China; and Center for Magnetic Resonance Research (Y.X., Y.S., M.M.K., K.C., X.J.Z.) and Departments of Radiology (K.C., T.M.A., X.J.Z.), Bioengineering (Y.S., X.J.Z.), and Neurosurgery (X.J.Z.), University of Illinois Hospital and Health Sciences System, Chicago, Illinois.

This work was supported, in part, by the National Natural Science Foundation of China (grant Nos. 81171308 and 81471230), the National Program of the Ministry of Science and Technology of China during the "12th Five-Year Plan" (grant No. 2011BAI08B10), and the US National Institutes of Health (grant No. 1S10RR028898). organs, including the brain, eye, kidney, heart, vasculature, and peripheral nerves due to long-term sustained hyperglycemia. Brain damage caused by diabetes has attracted increased attention during

Paper previously presented in part at: Annual Meeting of the International Society for Magnetic Resonance in Medicine, May 30 -June 5, 2015; Toronto, Ontario, Canada.

Please address correspondence to X. Joe Zhou, PhD, Advanced Imaging Center, Ste 103, 2242 W Harrison St, Chicago, IL 60612; e-mail: xjzhou@uic.edu; Jianzhi Wang, PhD, Department of Pathophysiology, Tongji Medical College, Huazhong University of Science and Technology, 13 Hangkong Rd, 430030, Wuhan, P.R. China; e-mail: wangjz@mail.hust.edu.cn

- Indicates open access to non-subscribers at www.ajnr.org

三 Indicates article with supplemental on-line table.

Indicates article with supplemental on-line photos.

http://dx.doi.org/10.3174/ajnr.A4740 
recent years. Longitudinal population-based studies have revealed that the relative risk of dementia is 1.2-2.8 times higher in individuals with diabetes relative to those without. ${ }^{2,3}$

Although neuropsychological tests such as the Mini-Mental State Examination and the Montreal Cognitive Assessment remain the prevailing methods for evaluating patients with T2DM with and without cognitive decline, a number of neuroimaging studies have emerged to investigate the compromised brain functions of patients with T2DM by measuring changes in perfusion, ${ }^{4}$ metabolism, ${ }^{5}$ spontaneous brain activity at resting state, ${ }^{6}$ and functional connectivity. ${ }^{7}$ The observed functional changes have been associated with sustained hyperglycemia and the resulting alterations in cerebral vasculature, neurotrophic factors, and neurotransmitters. ${ }^{8}$ To study the underlying brain structural changes associated with the functional and metabolic abnormalities, Zhang et $\mathrm{al}^{9}$ compared the gray matter volume of patients with T2DM with and without mild cognitive impairment (MCI) and concluded that middle temporal gyrus atrophy was associated with an increased risk of MCI in patients with T2DM. Cerebral atrophy and WM hyperintensities were also reported in patients with $\mathrm{T} 2 \mathrm{DM}^{10}$ and were linked to compromised cognition. ${ }^{11}$ Additionally, recent studies focusing on type 1 diabetes mellitus revealed WM changes in the superior parietal lobule ${ }^{12}$ and more pronounced WM hyperintensities in patients with slower information processing. ${ }^{13}$ The observed changes in WM are of particular interest, given its important role as a conduit to transmit neuronal signals to the functional units in the gray matter.

DTI is a powerful neuroimaging method to study WM structural changes in many neurologic disorders, including MCI and Alzheimer disease. ${ }^{14}$ In a study by Hsu et al, ${ }^{15}$ decreased fractional anisotropy (FA) was found in the frontal lobe of patients with T2DM with both global and voxel-based analyses. Decreased FA in the cingulum bundle and uncinate fasciculus, ${ }^{16}$ and increased mean diffusivity (MD) in the bilateral hemisphere tracts, ${ }^{17}$ were also reported. Recently, Zhang et $\mathrm{al}^{18}$ observed that patients with T2DM with various degrees of cognitive impairment exhibited widespread WM disruptions, which correlated well with executive dysfunction.

Although the risk of developing cognitive impairment is considerably elevated among patients with T2DM, not all patients with T2DM develop cognitive impairment based on neuropsychological tests. This raises an interesting and important question of whether the observed WM change occurs only in patients with T2DM with cognitive impairment or in all patients with T2DM. We hypothesize that the WM change in patients with T2DM, in accordance with cognitive decline, is a gradual and continuous process that may not be adequately reflected by neuropsychological test scores but can be captured by DTI. The present study, therefore, aimed to test this hypothesis by subdividing the patients with T2DM into 2 groups, those exhibiting MCI and those without, and investigating their DTI parameter changes against a third group of age-, sex-, and education-matched healthy subjects. Additionally, the study also intended to demonstrate that DTI parameters can be used quantitatively to complement neuropsychological test scores in characterizing patients with T2DM with and without MCI.

\section{MATERIALS AND METHODS \\ Subjects}

With approval by the institutional review board, we used a prospective cross-sectional study design with a recruitment of 70 right-handed adult subjects who provided written informed consent. Among the subjects, 44 were patients with T2DM (between 51 and 72 years of age; 27 women) recruited from the endocrinology clinic of Tongji Hospital of China between May 2013 and May 2014. Detailed information about hypoglycemic agent application, family history, and clinical symptoms and complications was collected. Clinical examinations and laboratory tests, including blood biochemistry; lipids and cholesterol levels; plasma glucose and glycosylated hemoglobin Alc levels; and body mass index, were performed.

Diagnosis of T2DM was based on established criteria (ie, diabetes symptoms and a fasting plasma glucose level of $>7.0$ $\mathrm{mmol} / \mathrm{L}$ or a random plasma glucose level of $>11.1 \mathrm{mmol} / \mathrm{L}$ or a 2-hour glucose level of $>11.1 \mathrm{mmol} / \mathrm{L}$ after an oral glucose tolerance test), according to the American Diabetes Association recommendations. ${ }^{19}$ A battery of neuropsychological tests was performed on the 44 patients with confirmed T2DM to assess their cognitive functions as detailed in the next subsection. On the basis of the results of the neuropsychological tests, the patients with T2DM were divided into 2 groups with and without cognitive impairment. Twenty-six subjects with euglycemia (between 50 and 73 years of age; 17 women; fasting glucose level of $<7.0$ $\mathrm{mmol} / \mathrm{L}$, glycosylated hemoglobin A1c percentage of $<6.0 \%$ ) with normal cognition (Montreal Cognitive Assessment score of $\geq 28$ and Mini-Mental State Examination score of $\geq 27$ ) were also enrolled to serve as age-, sex-, and education-matched healthy controls (denoted as the healthy control [HC] group hereafter).

For all 3 groups, we used the following exclusion criteria: 1) organic lesions in the brain, such as brain tumors, cerebral infarction, hemorrhage, or vascular malformation; 2) a history of stroke, epilepsy, head trauma, or brain surgery; 3 ) systemic organic disease or a history of tumors; 4) moderate and severe hypertension (systolic pressure of $\geq 160 \mathrm{~mm} \mathrm{Hg}$ or diastolic pressure of $\geq 100 \mathrm{~mm} \mathrm{Hg}$ ), or hyperlipidemia; 5) any contraindication to MR imaging examination, such as the presence of metallic implants, fixed metal dentures, pacemaker, or claustrophobia; or 6) other types of diabetes. In addition to the above criteria, 2 patients were excluded from data analysis because of excessive motion during the MR imaging $(n=1)$ or scanner malfunction $(n=1)$, resulting in 42 patients with T2DM and 26 healthy controls (no exclusions) whose imaging data were used in the final analysis.

\section{Cognitive Assessment}

All patients underwent comprehensive physical, neurologic, and neuropsychological assessments, which included the Mini-Mental State Examination, Montreal Cognitive Assessment, Hachinski Ischemic Score, Activities of Daily Living Test, and Auditory Verbal Learning Test, performed by 2 neurologists (Z.X. and Q.Z.), to subdivide the patients with T2DM into 2 groups with and without MCI. The inclusion criteria for the MCI group were the following: 1) memory decline; 2) both Montreal Cognitive Assessment and Mini-Mental State Examination scores of $\leq 27$; 
and 3) absence of any other physical or mental disorders that can lead to cognitive impairment, resulting in 20 patients with T2DM in the MCI group (the DM-MCI group). The remaining 22 patients formed the group with normal cognition (the DM-NC group). The Hachinski Ischemic Score and Activities of Daily Living tests were used to exclude vascular dementia and evaluate the daily life abilities. Consistency in age, sex, and education level among the DM-MCI, DM-NC, and HC groups was determined with a 1-way ANOVA and a $\chi^{2}$ test.

\section{Image Acquisition}

MR images were acquired on a 3T MR imaging scanner (Discovery MR750; GE Healthcare, Milwaukee, Wisconsin) by using a commercial 32-channel head coil. The subjects were padded with flexible foam to limit head motion. Using axial T2 FLAIR (TR/TE/ $\mathrm{TI}=8400 / 160 / 2100 \mathrm{~ms}$, section thickness $=5 \mathrm{~mm}$, section spacing $=1.5 \mathrm{~mm}$, matrix size $=256 \times 256, \mathrm{FOV}=24.0 \times 24.0 \mathrm{~cm}^{2}$, and NEX $=1)$ and a sagittal T1-weighted 3D brain volume imaging sequence $\left(\mathrm{TR} / \mathrm{TE} / \mathrm{TI}=8.2 / 3.2 / 450 \mathrm{~ms}\right.$, flip angle $=12^{\circ}$, section thickness $=1 \mathrm{~mm}$, matrix size $=256 \times 256 \times 160$, FOV $=$ $25.6 \times 25.6 \mathrm{~cm}^{2}$, and NEX $=1$ ), we obtained high-resolution anatomic images to exclude possible lesions specified in the exclusion criteria. Following anatomic imaging, DTI data were obtained in the axial plane by using a single-shot diffusion-weighted echo-planar imaging sequence with the following parameters: $\mathrm{TR} / \mathrm{TE}=8500 / 66.3 \mathrm{~ms}, \mathrm{FOV}=25.6 \times 25.6 \mathrm{~cm}^{2}$, matrix size $=$ $128 \times 128$, section thickness $=2 \mathrm{~mm}$, number of sections $=70$, number of diffusion gradient directions $=64$, b-value $=1000$ $\mathrm{s} / \mathrm{mm}^{2}$, number of images at a b-value of $0 \mathrm{~s} / \mathrm{mm}^{2}=5$, acceleration factor $=2$, and scan time $=9$ minutes 55 seconds.

\section{fMRI of the Brain Software Library and Tract-Based Spatial Statistics}

The diffusion tensor images were processed by using the fMRI of the Brain Software Library (http://www.fmrib.ox.ac.uk/fsl), or FSL. ${ }^{20}$ Voxelwise statistical analysis of the images was performed by using Tract-Based Spatial Statistics (TBSS; http://fsl.fmrib. ox.ac.uk/fsl/fslwiki/TBSS) $)^{21}$ with the following steps: First, brain was extracted by using the Brain Extraction Tool (http://fsl.fmrib. ox.ac.uk/fsl/fslwiki/BET). An FSL "eddy" tool (http://fsl.fmrib. ox.ac.uk/fsl/fslwiki/eddy) was applied as a preventive measure to reduce inconsistent image distortion. After generating the FA maps by using the FMRIB Diffusion Toolbox (http://fsl.fmrib.ox. ac.uk/fsl/fslwiki/FDT), we aligned the images from all subjects to an FA standard template through a nonlinear coregistration. The aligned FA maps were then averaged to produce a group mean image, which was used to generate an FA skeleton highlighting the tracts common to the entire group. For each subject, an FA threshold of 0.2 was used before projecting the aligned FA map onto this skeleton. The resulting skeletonized FA maps were then fed into a voxelwise group-level analysis. ${ }^{22}$ In addition to FA, diffusivity maps based on MD, axial diffusivity $\left(\lambda_{1}\right.$, the principal eigenvalue), and radial diffusivity $\left(\lambda_{23}\right.$, the average of the 2 remaining eigenvalues) were generated by using the same steps outlined above. Using an FSL permutation test (FSL Randomise tool with 500 permutations; http://fsl.fmrib.ox.ac.uk/fsl/fslwiki/ Randomise), we tested FA, MD, $\lambda_{1}$, and $\lambda_{23}$ for differences be- tween the means of the DM-MCI, DM-NC, and HC groups. A significance level of $P<.05$ was used for each of the 4 DTI parameters (FA, MD, $\lambda_{1}$, and $\lambda_{23}$ ) to declare differences among the patient groups.

\section{ROI-Based Quantitative Analysis}

The Johns Hopkins University WM tractography atlas ${ }^{23}$ in FSL was used as a standard for WM parcellation. The entire WM was parceled into 48 ROIs by using the 1-mm Johns Hopkins University-ICBM labels (http://fsl.fmrib.ox.ac.uk/fsl/fslwiki/Atlases). Specific fiber tracts reported to be relevant to MCI in previous studies ${ }^{24-26}$ were selected in the telencephalon as well as 4 regions in the cerebellum and the brain stem (Fig 1). FA and MD were calculated by averaging the pixel values in each ROI and were reported as mean \pm SD. Using SPSS 17.0 software (IBM, Armonk, New York), we performed a receiver operating characteristic (ROC) analysis to determine the area under the ROC curve for assessing the performance of separating the DM-MCI and DM-NC groups when FA and MD of the right cingulum (hippocampus) were used individually or in combination via a logistic regression algorithm.

The ROC analysis for the combination of FA and MD was performed by using a binomial (bivariate) logistic regression with patient condition (DM-MCI or DM-NC) as the dichotomous criterion variable and FA and $\mathrm{MD}$ as the dichotomous predictor variables. This method estimated the probability $\left(P_{0}\right)$ of being in the DM-MCI group by using the following logistic function:

$$
P_{0}=\exp \left(a_{0}+a_{1} F A+a_{2} M D\right) /\left[1+\exp \left(a_{0}+a_{1} F A+a_{2} M D\right)\right],
$$

where $a_{0}$ is a constant and $a_{1}$ and $a_{2}$ are the regression coefficients for FA and MD, respectively. The regression coefficients were determined by using a maximum likelihood method. ${ }^{27}$

\section{RESULTS}

\section{Clinical Data}

The clinical and neuropsychological characteristics of the 3 subject groups are summarized in the On-line Table. No significant difference was observed among the 3 groups in age $(P=.130)$, sex $(P=.854)$, years of education $(P=.216)$, and body mass index $(P=.291)$. The DM-MCI group exhibited a higher level of glycosylated hemoglobin A1c $(P=.008)$ and a trend toward a significant increase in disease duration $(P=.066)$ compared with the DM-NC group.

\section{Whole-Brain DTI Comparisons among Groups}

The whole-brain TBSS analysis revealed that 7.3\% (10,102/137,832 voxels) and $24.9 \%(34,353 / 137,832$ voxels $)$ of the parcellated regions exhibited decreased FA and increased $\mathrm{MD}$, respectively, in the DM-MCI group compared with the DM-NC group (Fig 2). Analysis of the individual eigenvalues illustrated that the reduced FA and increased MD in the DM-MCI group were caused primarily by an elevated radial diffusivity $\left(\lambda_{23}\right)$, rather than changes in axial diffusivity $\left(\lambda_{1}\right)$ (Fig 2). Compared with the results in Fig 2, more extensive changes in FA (36.6\%; 50,384/137,832 voxels) and $\operatorname{MD}(58.8 \% ; 81,104 / 137,832$ voxels) of the WM regions across the entire brain were observed between the DM-MCI and the HC 


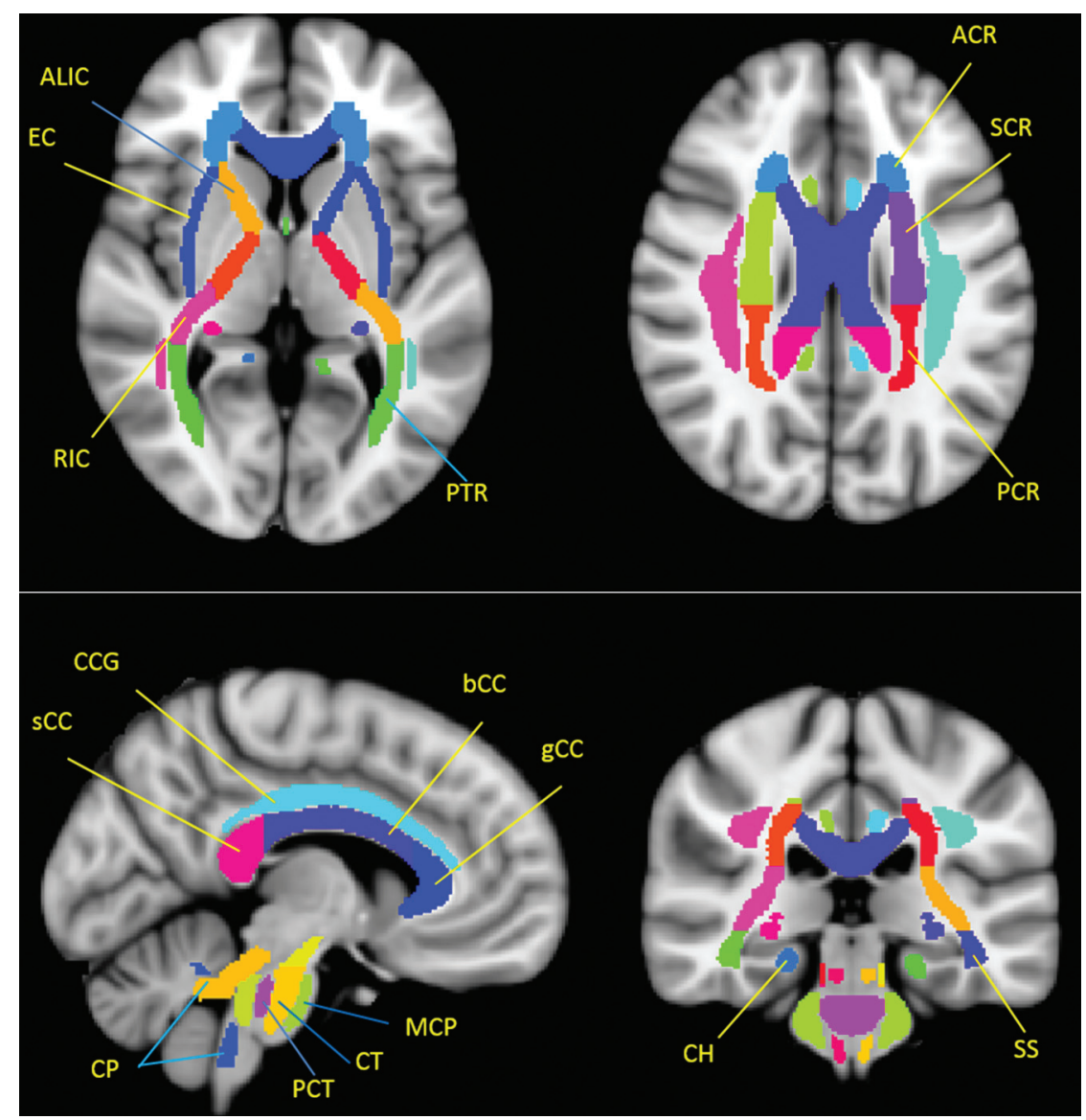

FIG 1. Selected ROIs in the individual fiber analysis according to a Johns Hopkins University-ICBM labels 1-mm template. ".R and .L" in the text indicate right and left side, respectively. In the telencephalon, ALIC indicates anterior limb of internal capsule; ACR, anterior corona radiata; $\mathrm{g} / \mathrm{b} / \mathrm{s}$ CC, corpus callosum (genu/body/splenium); CCG, cingulum (cingulate gyrus); $\mathrm{CH}$, cingulum (hippocampus); EC, external capsule; PCR, posterior corona radiata; PTR, posterior thalamic radiation (including optic radiation); RIC, retrolenticular part of internal capsule; SCR, superior corona radiata; SS, sagittal striatum (including the inferior longitudinal fasciculus and inferior frontooccipital fasciculus). In the cerebellum and brain stem, CP, indicates cerebellar peduncle; CT, corticospinal tract; MCP, middle cerebellar peduncle; and $\mathrm{PCT}$, pontine crossing tract (a part of $\mathrm{MCP})$.

groups (Fig 3). In addition, $\lambda_{1}$ also showed noticeable changes in Fig 3. These observations indicate that DTI parameters of the DM-NC group differed from those of the HC group; this difference suggests that gradual WM changes have already occurred in the patients with DM-NC, even when neuropsychological tests did not indicate MCI. These findings support our hypothesis. Additional evidence to reinforce our hypothesis can be found in Fig 4, where a direct comparison between the DM-NC and the HC groups reveals moderate changes in radial diffusivity $\left(\lambda_{23}\right)$ in several regions, including the bilateral external capsule and the temporal WM areas, as well as the right frontal WM areas and corona radiata.

\section{Differences in FA and MD of Specific Fiber Tracts among the Groups}

Tables 1 and 2 summarize the FA and MD differences $(P<.05$ with false discovery rate correction), respectively, in the selected fibers across the 3 groups. Among the 15 fiber tracts analyzed by using the Johns Hopkins University atlas, ${ }^{23}$ significantly decreased FA and increased MD were observed in the left external capsule between the DMMCI and DM-NC groups. Additionally, we also observed FA differences in the left anterior limb of the internal capsule, right and left anterior corona radiata, left posterior thalamic radiation, and right and left cingulum (hippocampus), and MD differences in the left retrolenticular part of internal capsule, left superior corona radiata, and right sagittal striatum. These differences, which suggest that FA and MD may serve as alternative surrogates to the conventional neuropsychological test scores, are illustrated in Fig $5 A$ (for FA), $-B$ (for MD). More important, when the DM-NC and the $\mathrm{HC}$ groups were compared, significant differences in FA were found in the right corticospinal tract and right cerebral peduncle, and differences in MD were found in the right retrolenticular part of internal capsule and right external capsule. Again, these observations support our hypothesis. Overall, the atlas-based analyses on individual fiber tracts suggested that pronounced FA reduction and/or MD elevation occurred mainly in the internal/external capsule, corona radiata, and cingulum (in the vicinity of the hippocampus) regions.

\section{ROC Analysis}

The feasibility of using FA and MD to separate the DM-MCI and DM-NC groups, as indicated by Fig 5, is further demonstrated in an ROC analysis. Figure $6 \mathrm{~A}$ shows the ROC curves in the right cingulum (hippocampus), using DTI parameters for distinguishing DM-MCI (positive) and DM-NC (negative). FA (area under the ROC curve $=0.832 ; 95 \%$ CI, 0.705-0.958) had a higher area under the ROC curve than MD $(0.753 ; 95 \% \mathrm{CI}, 0.608-0.899)$. When we combined FA and MD by using a logistic regression model, the area under the ROC curve was further improved to 0.857 (95\% CI, 0.7350.979). Figure $6 B$ shows a scatterplot with all data of patients with T2DM. The best cutoff values of FA and MD, determined by using a Youden index, are indicated by the vertical and the horizontal dashed lines, respectively. The black dashed line corresponds to the cutoff probability of the best sensitivity (0.864) and specificity (0.800) when combining FA and MD.

\section{DISCUSSION}

The results of this study (Figs 2-4 and Tables 1 and 2) provided evidence to support our hypothesis that the WM change in patients with T2DM is a gradual and continuous process that may not be adequately reflected by neuropsychological test scores but can be captured in DTI parameters. The observations were made 


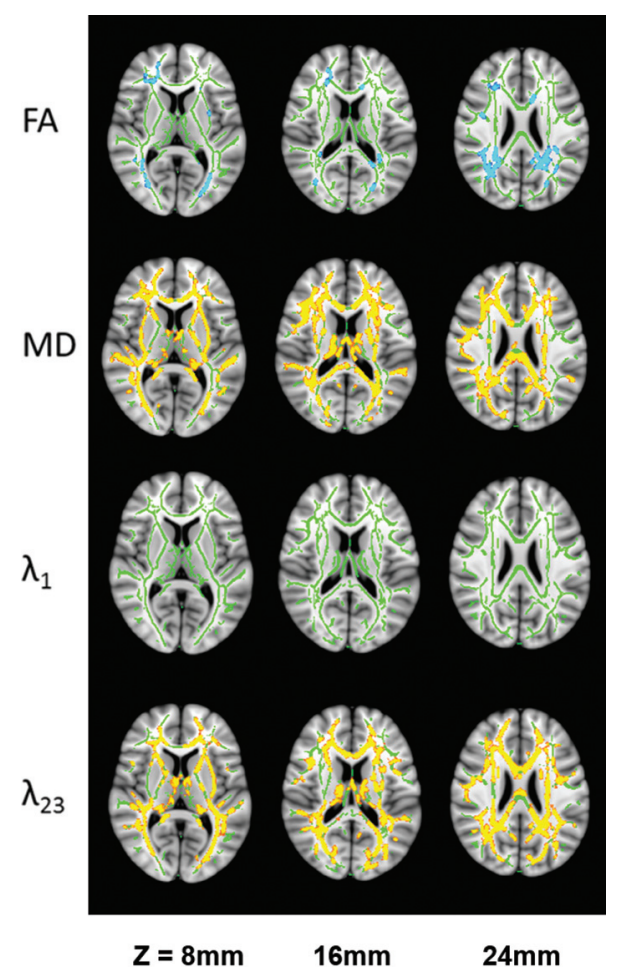

FIG 2. Differences in FA, MD, axial diffusivity $\left(\lambda_{1}\right)$, and radial diffusivity $\left(\lambda_{23}\right)$ between the DM-MCl and the DM-NC groups in 3 representative sections (see On-line Fig 1 for a complete set of images with wholebrain coverage). All results were obtained from an FSL TBSS analysis. A Montreal Neurological Institute-152 T1 1-mm brain standard space was used as a background image (gray-scale). Green indicates the FA skeleton with a threshold of 0.2 highlighting the fibers used in the comparison; blue-light-blue (thickened for better visibility), regions with decreased FA, MD, $\lambda_{1}$, or $\lambda_{23}$; and red-yellow (thickened for better visibility), regions with increased FA, MD, $\lambda_{1}$, or $\lambda_{23}$.

possible by dividing the patients with T2DM into 2 groups according to their cognitive functions and examining changes in DTI metrics in each group against healthy controls. Using a prevalent DTI analysis tool, FSL, we observed not only decreased FA and increased MD in the brains of the patients with T2DM with clinically confirmed MCI compared with those without (Figs 2 and 5), but also subtle yet important changes in radial diffusivity $\left(\lambda_{23}\right)$ between the DM-NC and the HC groups (Fig 4). These results have not been reported previously, to our knowledge. We also observed widespread FA and MD changes between the DMMCI and the HC groups (Fig 3), which reinforced the findings in a recent study. ${ }^{18}$

The widespread WM differences in Fig 2 indicate that the observed changes in DTI metrics are strongly coupled with cognitive decline. Using individual eigenvalues, we were able to determine that the FA and MD changes were associated with elevated radial diffusivity, instead of decreased axial diffusivity. Previous studies have related elevated radial diffusivity to a compromised myelin sheath and reduced axial diffusivity to axonal damage. ${ }^{28,29}$ Thus, the WM changes in Fig 2 are likely caused by changes in the myelin sheath or increased interstitial space between the myelin-covered axons in patients with DM-MCI. The exact mechanism explaining why long-term sustained hyperglycemia would compromise the myelin sheath remains unclear. Small-vessel alterations asso-

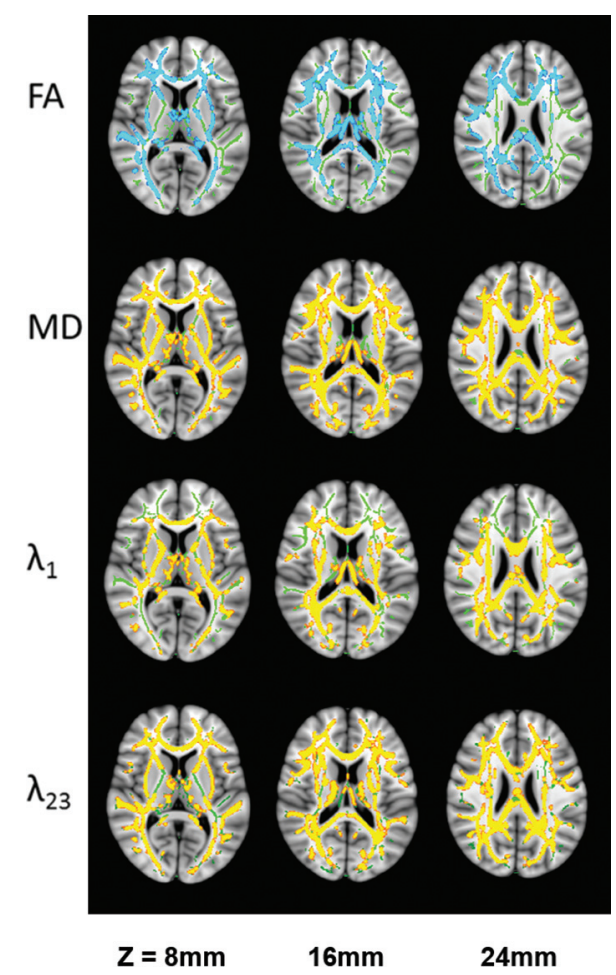

FIG 3. Group differences in $F A, M D$, axial diffusivity $\left(\lambda_{1}\right)$, and radial diffusivity $\left(\lambda_{23}\right)$ between the $\mathrm{DM}-\mathrm{MCl}$ and the $\mathrm{HC}$ groups in 3 representative sections (see On-line Fig 2 for a complete set of images with whole-brain coverage). All other details are the same as in the legend for Fig 2.

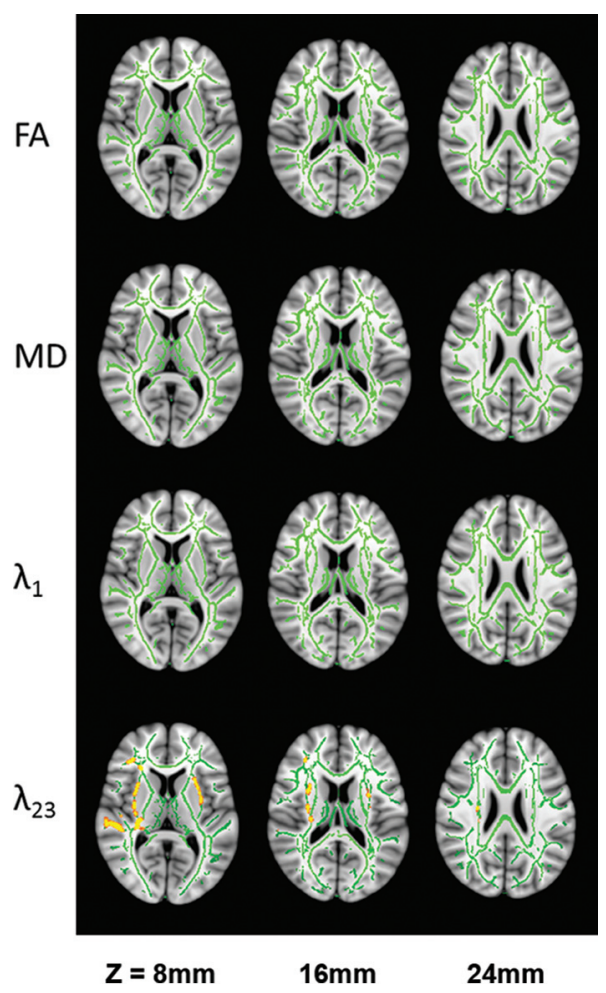

FIG 4. Group differences in FA, MD, axial diffusivity $\left(\lambda_{1}\right)$, and radial diffusivity $\left(\lambda_{23}\right)$ between the DM-NC and the HC groups in 3 representative sections (see On-line Fig 3 for a complete set of images with whole-brain coverage). All other details are the same as in the legend for Fig 2. 
Table 1: FA values in specific fiber tracts with significant differences among groups $(P<.05)$

\begin{tabular}{lcccc}
\hline \multicolumn{1}{c}{ JHU WM } & & & & \\
\hline ALIC.L & $0.595 \pm 0.025$ & $0.610 \pm 0.021$ & & .044 \\
ACR.R & $0.472 \pm 0.024$ & $0.487 \pm 0.019$ & & .035 \\
ACR.L & $0.471 \pm 0.029$ & $0.488 \pm 0.018$ & & .022 \\
PTR.L & $0.620 \pm 0.038$ & $0.641 \pm 0.027$ & & .045 \\
EC.L & $0.478 \pm 0.020$ & $0.490 \pm 0.017$ & & .043 \\
CH.R & $0.585 \pm 0.040$ & $0.616 \pm 0.024$ & & .004 \\
CH.L & $0.579 \pm 0.027$ & $0.599 \pm 0.026$ & & .019 \\
CT.R & & $0.548 \pm 0.030$ & $0.569 \pm 0.017$ & .003 \\
CP.R & & $0.590 \pm 0.021$ & $0.608 \pm 0.019$ & .002 \\
\hline
\end{tabular}

Note:-R indicates right; L, left; ALIC, anterior limb of internal capsule; ACR, anterior corona radiata; $\mathrm{CH}$, cingulum (hippocampus); EC, external capsule; PTR, posterior thalamic radiation (including the optic radiation); $C P$, cerebellar peduncle; $C T$, corticospinal tract; JHU, Johns Hopkins University.

Table 2: MD values $\left(\times 10^{-3} \mathrm{~mm}^{2} / \mathrm{s}\right)$ in specific fiber tracts with significant differences among groups $(P<.05)$

\begin{tabular}{lcccc}
\hline \multicolumn{1}{c}{ JHU WM } & & & & \\
\hline RIC.L & MD (DM-MCI) & MD (DM-NC) & MD (HC) & $P$ Value \\
SCR.L & $0.780 \pm 0.026$ & $0.760 \pm 0.029$ & & .031 \\
SS.R & $0.732 \pm 0.029$ & $0.712 \pm 0.026$ & & .021 \\
EC.L & $0.758 \pm 0.037$ & $0.815 \pm 0.030$ & & .024 \\
RIC.R & & $0.739 \pm 0.026$ & & .030 \\
EC.R & & $0.773 \pm 0.030$ & $0.755 \pm 0.021$ & .021 \\
\hline
\end{tabular}

Note:- R indicates right; L, left; RIC, the retrolenticular part of internal capsule; SCR, superior corona radiata; SS, sagittal striatum (including the inferior longitudinal fasciculus and inferior fronto-occipital fasciculus); EC, external capsule; JHU, Johns Hopkins University.

ciated with hypoperfusion and inflammatory factors can be possible causes. ${ }^{30}$

Although both Figs 2 and 3 compare the DM-MCI group against subjects without clinically confirmed cognitive impairment, the results are considerably different. In addition to changes in radial diffusivity, Fig 3 also shows extensive changes in axial diffusivity $\left(\lambda_{1}\right)$, suggesting axonal damage in patients with DM-MCI compared with HC. A number of studies have shown that T2DM and Alzheimer disease share several pathogeneses, including insulin deficit, glucose-mediated toxicity, and amyloid- $\beta$ peptide accumulation. ${ }^{8,31}$ One of the important pathologic bases of Alzheimer disease is neurofibrillary tangles, ${ }^{32}$ caused by the phosphorylated $\tau$ protein. As a terminal axonal lesion, neurofibrillary tangles and subsequent synapse loss can lead to progressive loss of memory and compromised cognition. The similar mechanism may explain the observed $\lambda_{1}$ elevation in Fig 3. More important, the increase in radial diffusivity in the patients with DM-NC shown in Fig 4 can be a significant finding because it suggests minor-to-moderate damage to the myelin sheath in the early phase of cognitive decline (eg, amnesia) in patients with T2DM before they can be clinically diagnosed by using neuropsychological test scores.

Our investigation of regional WM alterations was focused on the telencephalon because of its pivotal role in cognition and dementia. Not surprising, DTI parameter changes were observed in the cingulum near the hippocampus, which is related to learning and memory, ${ }^{24}$ as well as in the anterior limb and the retrolenticular area of the internal capsule. Furthermore, the FA or MD differences between the DM-NC and HC groups in the corticospinal tract and external capsule (Tables 1 and 2) provide more
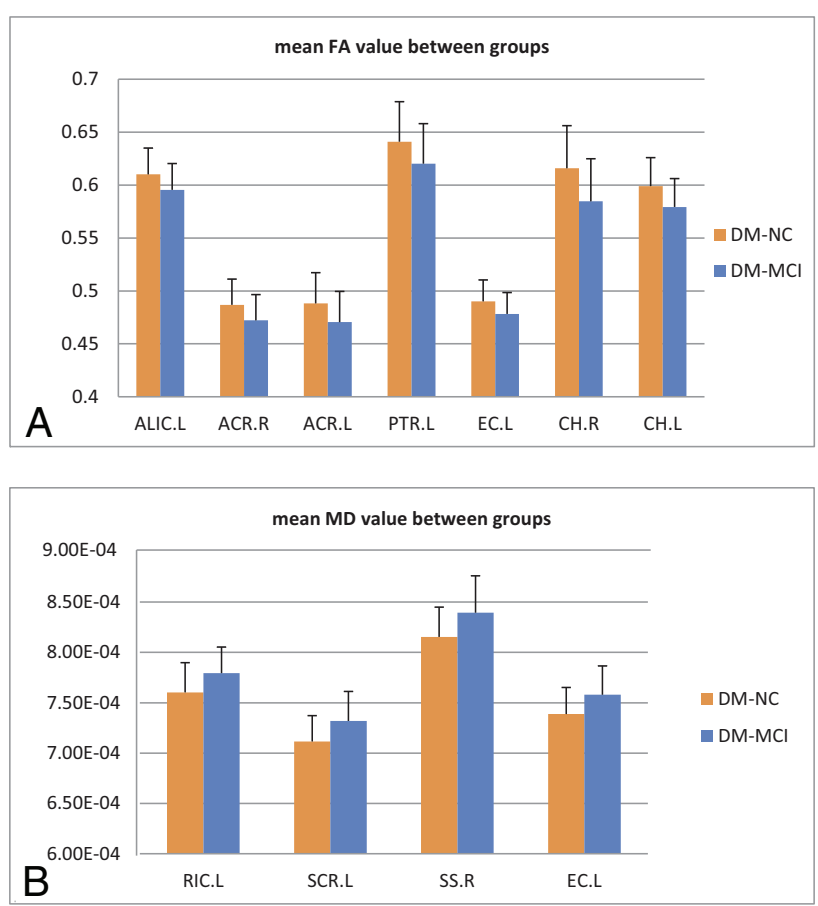

FIG 5. Differences in $F A(A)$ and $M D(B)$ values for specific fiber tracts in an atlas-based ROI analysis between the DM-MCl (blue) and the DM-NC (orange) groups. All fibers in the figure show a statistically significant difference $(P<.05)$. The bar height indicates the mean, and the line on top of the bar represents the SD. L indicates left; R, right; ALIC, anterior limb of internal capsule; ACR, anterior corona radiata; $\mathrm{CH}$, cingulum (hippocampus); EC, external capsule; PTR, posterior thalamic radiation (including the optic radiation); RIC, retrolenticular part of internal capsule; SCR, superior corona radiata; SS, sagittal striatum (including the inferior longitudinal fasciculus and inferior fronto-occipital fasciculus).

specific evidence to support our hypothesis that early changes during the course of cognitive decline can be detected by DTI before the patients become symptomatic.

The present study has also demonstrated that FA and MD can become potential imaging markers (Fig 6) that are sensitive to cognitive declines in patients with T2DM. These markers can be used individually or combined to complement the existing neuropsychological tests. Zhang et $\mathrm{al}^{18}$ recently reported that FA changes in the external capsule were correlated with executive dysfunction. Reijmer et $\mathrm{al}^{17}$ observed that increased MD was associated with slowing information processing and worsened memory performance. These studies, together with our results, all indicate that FA and MD can quantitatively characterize the cognitive decline process, which would eventually lead to clinical symptoms to be reflected in the neuropsychological scores.

Our study has several limitations. First, despite the attempt to exclude other causes of WM changes, it is still possible that an underlying process independent of diabetes mellitus is associated with the cognitive decline. Patients without diabetes with similar cognition need to be studied to control for the possibility that the cognitive decline rather than diabetes mellitus is associated with changes in DTI measures. Nonetheless, the present study suggests that DTI metrics have the potential to predict cognitive impairment in patients with T2DM (Fig 6). Second, although we excluded subjects with severe and moderate hypertension or hyper- 

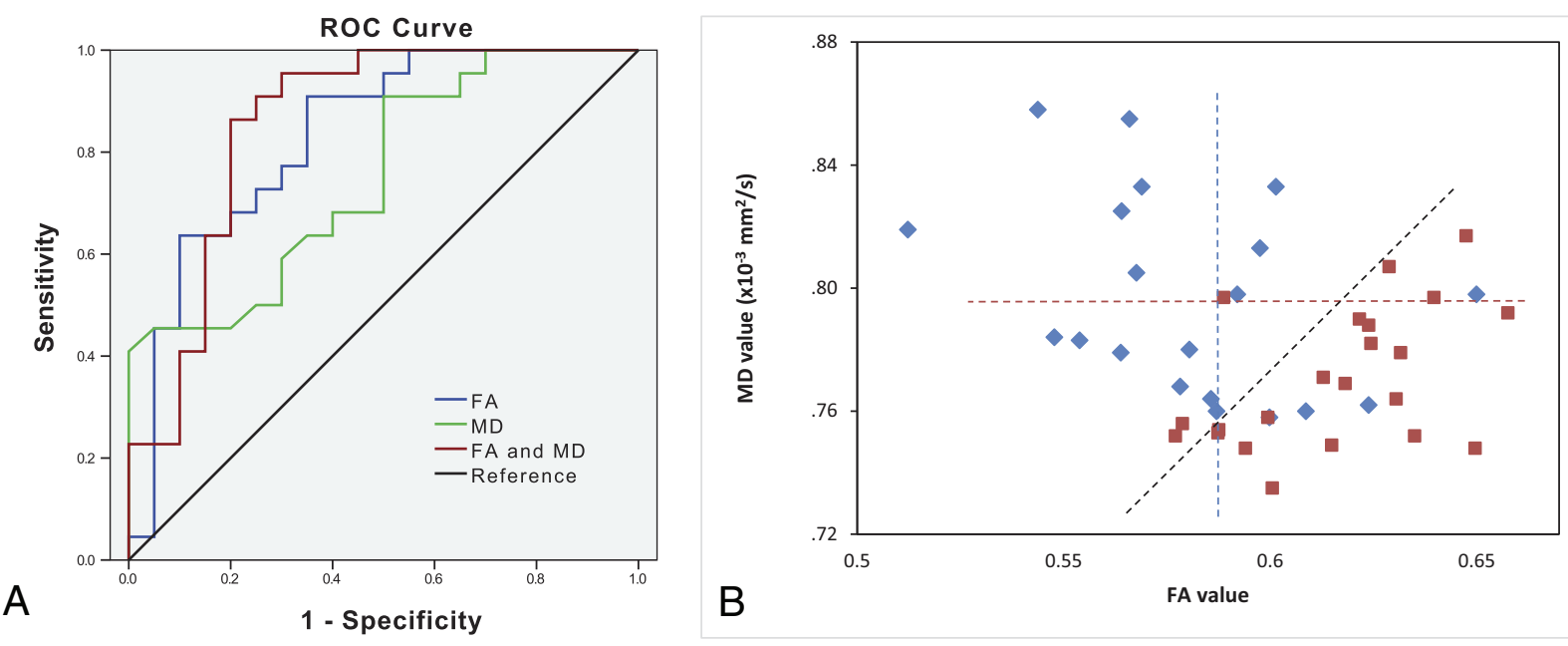

FIG 6. $A$, ROC curves for FA (blue), MD (green), and a combination of FA and MD (red) of the right cingulum (hippocampus) for separating $\mathrm{DM}-\mathrm{MCl}$ and DM-NC patients. B, Scatterplots of FA versus MD for all patients with DM-NC (red) and DM-MCI (blue). The dashed lines indicate the cutoff values for FA (blue), MD (red), and the combination of FA and MD (black). The black dashed line corresponds to a sensitivity of 0.864 and a specificity of 0.800 .

lipidemia, a small number of subjects with mild hypertension or hyperlipidemia were included to increase the statistical power. We observed very similar results in separate analyses with and without the inclusion of subjects with mild hypertension (or hyperlipidemia), indicating that the effect caused by mild hypertension or hyperlipidemia was not substantial. Third, because rigorous clinical diagnosis of MCI remains a challenge, dividing the patients with T2DM into the DM-MCI and DM-NC groups can be subject to inaccuracy. Last, given the slow development of MCI, a longitudinal study would be needed to test whether DTI measures can predict the development of cognitive impairment in patients with T2DM.

\section{CONCLUSIONS}

Our study has demonstrated that DTI parameters can show a significant difference between patients with T2DM with and without MCI, suggesting their potential role as an imaging marker for detecting cognitive decline in patients with T2DM. More important, our study also indicates that the DTI parameters may capture gradual and continuous WM changes that can be associated with early stages of cognitive decline in patients with T2DM before they can be diagnosed clinically by using the conventional neuropsychological test scores.

\section{ACKNOWLEDGMENTS}

The authors are grateful to Dr Keith R. Thulborn for helpful discussions and to Dr Winnie Mar for proofreading the manuscript.

Disclosures: Yi Sui-UNRELATED: Consultancy: GE Healthcare. Xiaohong Joe Zhou-RELATED: Grant: National Institutes of Health*; UNRELATED: Consultancy: Horizon Medical Physics Services; Grants/Grants Pending: AbbVie Pharmaceuticals*; Royalties: Elsevier Publishing; Stock/Stock Options: Apple, Google. *Money paid to the institution.

\section{REFERENCES}

1. Whiting DR, Guariguata L, Weil C, et al. IDF Diabetes Atlas: global estimates of the prevalence of diabetes for 2011 and 2030. Diabetes Res Clin Pract 2011;94:311-21 CrossRef Medline
2. McCrimmon RJ, Ryan CM, Frier BM. Diabetes and cognitive dysfunction. Lancet 2012;379:2291-99 CrossRef Medline

3. Biessels GJ, Staekenborg S, Brunner E, et al. Risk of dementia in diabetes mellitus: a systematic review. Lancet Neurol 2006;5:64-74 CrossRef Medline

4. Rusinek H, Ha J, Yau PL, et al. Cerebral perfusion in insulin resistance and type 2 diabetes. J Cereb Blood Flow Metab 2015;35:95-102 CrossRef Medline

5. Sahin I, Alkan A, Keskin L, et al. Evaluation of in vivo cerebral metabolism on proton magnetic resonance spectroscopy in patients with impaired glucose tolerance and type 2 diabetes mellitus. J Diabetes Complications 2008;22:254-60 CrossRef Medline

6. Cui Y, Jiao Y, Chen YC, et al. Altered spontaneous brain activity in type 2 diabetes: a resting-state functional MRI study. Diabetes 2014; 63:749-60 CrossRef Medline

7. Musen G, Jacobson AM, Bolo NR, et al. Resting-state brain functional connectivity is altered in type 2 diabetes. Diabetes 2012;61: 2375-79 CrossRef Medline

8. Umegaki $\mathrm{H}$. Type 2 diabetes as a risk factor for cognitive impairment: current insights. Clin Interv Aging 2014;9:1011-19 CrossRef Medline

9. Zhang $\mathrm{Y}$, Zhang X, Zhang J, et al. Gray matter volume abnormalities in type 2 diabetes mellitus with and without mild cognitive impairment. Neurosci Lett 2014;562:1-6 CrossRef Medline

10. de Bresser J, Tiehuis AM, van den Berg E, et al; Utrecht Diabetic Encephalopathy Study Group. Progression of cerebral atrophy and white matter hyperintensities in patients with type 2 diabetes. Diabetes Care 2010;33:1309-14 CrossRef Medline

11. Moran C, Phan TG, Chen J, et al. Brain atrophy in type 2 diabetes: regional distribution and influence on cognition. Diabetes Care 2013;36:4036-42 CrossRef Medline

12. Antenor-Dorsey JA, Meyer E, Rutlin J, et al. White matter microstructural integrity in youth with type 1 diabetes. Diabetes 2013;62: 581-89 CrossRef Medline

13. Nunley KA, Ryan CM, Orchard TJ, et al. White matter hyperintensities in middle-aged adults with childhood-onset type 1 diabetes. Neurology 2015;84:2062-69 CrossRef Medline

14. Stahl R, Dietrich O, Teipel SJ, et al. White matter damage in Alzheimer disease and mild cognitive impairment: assessment with diffusion-tensor MR imaging and parallel imaging techniques. Radiology 2007;243:483-92 CrossRef Medline

15. Hsu JL, Chen YL, Leu JG, et al. Microstructural white matter abnor- 
malities in type 2 diabetes mellitus: a diffusion tensor imaging study. Neuroimage 2012;59:1098-105 CrossRef Medline

16. Hoogenboom WS, Marder TJ, Flores VL, et al. Cerebral white matter integrity and resting-state functional connectivity in middle-aged patients with type 2 diabetes. Diabetes 2014;63:728-38 CrossRef Medline

17. Reijmer YD, Brundel M, de Bresser J, et al; Utrecht Vascular Cognitive Impairment Study Group. Microstructural white matter abnormalities and cognitive functioning in type 2 diabetes: a diffusion tensor imaging study. Diabetes Care 2013;36:137-44 CrossRef Medline

18. Zhang J, Wang Y, Wang J, et al. White matter integrity disruptions associated with cognitive impairments in type 2 diabetic patients. Diabetes 2014;63:3596-605 CrossRef Medline

19. American Diabetes Association. Diagnosis and classification of diabetes mellitus. Diabetes Care 2013;36(suppl 1):S67-74 CrossRef Medline

20. Jenkinson M, Beckmann CF, Behrens TE, et al. FSL. Neuroimage 2012;62:782-90 CrossRef Medline

21. Smith SM, Jenkinson M, Johansen-Berg H, et al. Tract-based spatial statistics: voxelwise analysis of multi-subject diffusion data. Neuroimage 2006;31:1487-505 CrossRef Medline

22. Smith SM, Johansen-Berg H, Jenkinson M, et al. Acquisition and voxelwise analysis of multi-subject diffusion data with tract-based spatial statistics. Nat Protoc 2007;2:499-503 CrossRef Medline

23. Mori S, Oishi K, Jiang $\mathrm{H}$, et al. Stereotaxic white matter atlas based on diffusion tensor imaging in an ICBM template. Neuroimage 2008;40:570-82 CrossRef Medline
24. Seib DR, Martin-Villalba A. Neurogenesis in the normal ageing hippocampus: a mini-review. Gerontology 2015;61:327-35 CrossRef Medline

25. Chua TC, Wen W, Slavin MJ, et al. Diffusion tensor imaging in mild cognitive impairment and Alzheimer's disease: a review. Curr Opin Neurol 2008;21:83-92 CrossRef Medline

26. Liu Y, Spulber G, Lehtimäki KK, et al. Diffusion tensor imaging and tract-based spatial statistics in Alzheimer's disease and mild cognitive impairment. Neurobiol Aging 2011;32:1558-71 CrossRef Medline

27. Menard SW. Applied Logistic Regression Analysis. Thousand Oaks, California: Sage; 2002:1-120

28. Neil JJ. Diffusion imaging concepts for clinicians. J Magn Reson Imaging 2008;27:1-7 CrossRef Medline

29. Song SK, Sun SW, Ramsbottom MJ, et al. Dysmyelination revealed through MRI as increased radial (but unchanged axial) diffusion of water. Neuroimage 2002;17:1429-36 CrossRef Medline

30. Peterson JW, Bö L, Mörk S, et al. Transected neurites, apoptotic neurons, and reduced inflammation in cortical multiple sclerosis lesions. Ann Neurol 2001;50:389-400 CrossRef Medline

31. Biessels GJ, Deary IJ, Ryan CM. Cognition and diabetes: a lifespan perspective. Lancet Neurol 2008;7:184-90 CrossRef Medline

32. Butterfield DA, Di Domenico F, Barone E. Elevated risk of type 2 diabetes for development of Alzheimer disease: a key role for oxidative stress in brain. Biochim Biophys Acta 2014;1842:1693-706 CrossRef Medline 